Acta Crystallographica Section B

Structural

Science

ISSN 0108-7681

Editor: Carolyn P. Brock

\title{
The structure of benzoin
}

\author{
M. Haisa, S. Kashino and M. Morimoto
}

This electronic document was scanned from an archival copy of material deposited to accompany a paper published in an IUCr journal. In many cases the only accessible copy was a microfilm of a poor-quality original. 
$(1)$

Observed and calculated structure factors (ail) for benzoin

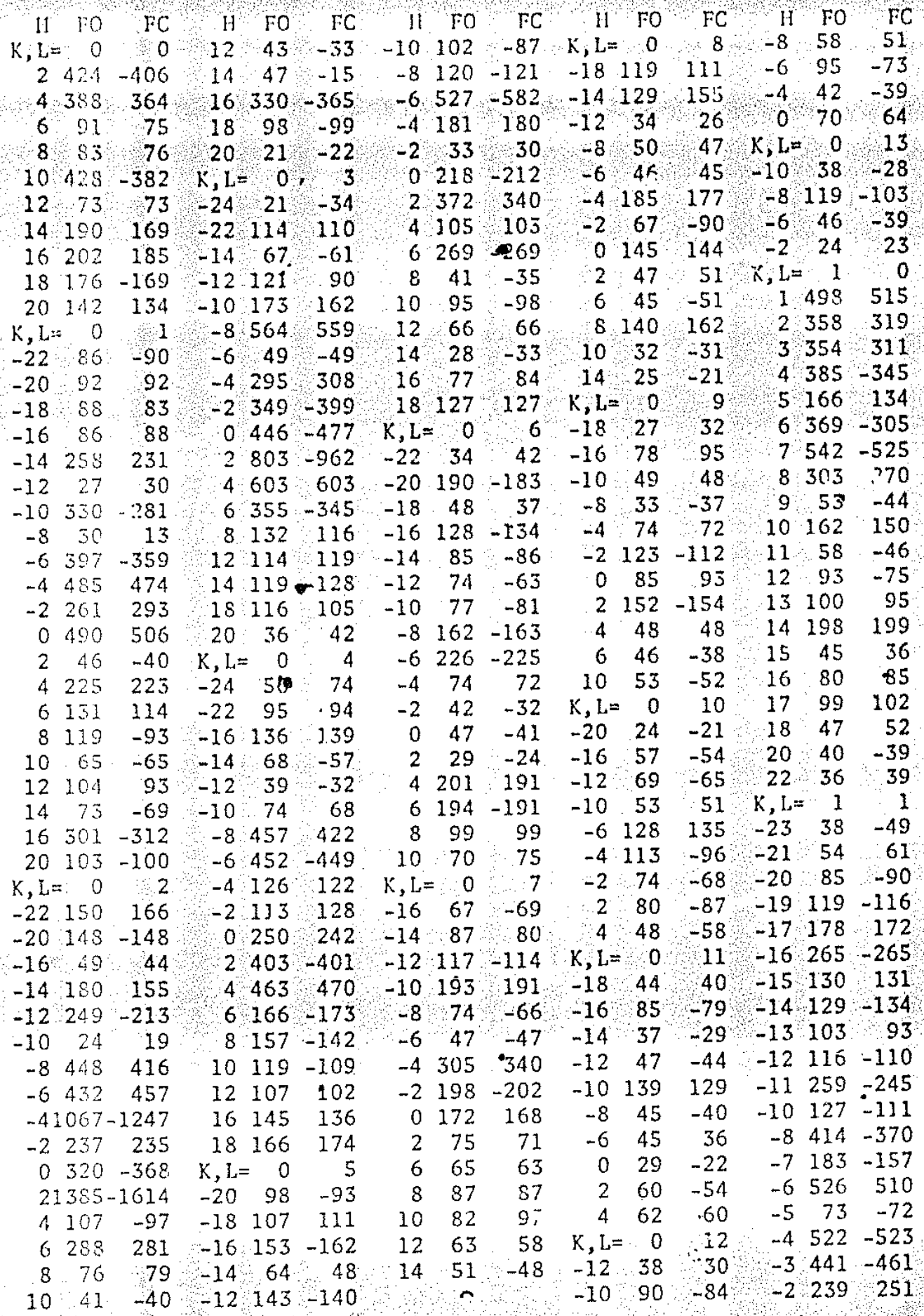




\section{(2)}

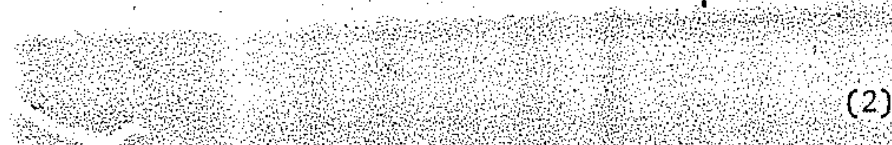

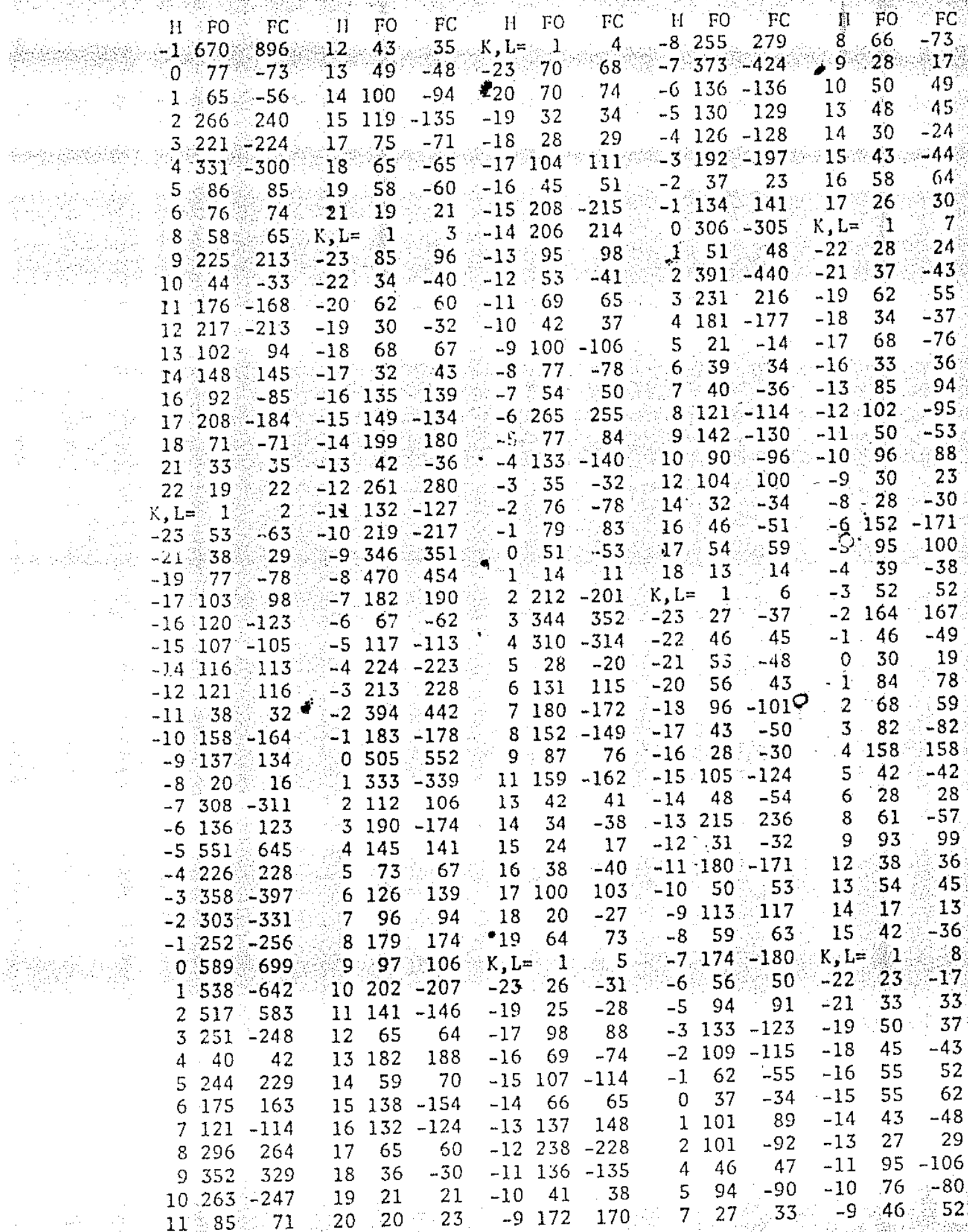




\begin{tabular}{|c|c|c|c|c|c|c|c|c|c|c|c|c|c|c|}
\hline \} & & & $n_{3}$ & & & & & & & & & & & \\
\hline & & 186 & -4 & 32 & -30 & 12 & 108 & -99 & -15 & 116 & & -3 & 335 & \\
\hline-7 & 45 & -44 & -3 & & -30 & 13 & 37 & 34 & -14 & 114 & -123 & 2 & & \\
\hline- & & -48 & -2 & 3 & 28 & 14 & & & -11 & 27 & & -1 & 29 & \\
\hline & 114 & 13 & & 3 & $-3 i$ & & & & -10 & & & & 225 & \\
\hline-3 & 73 & -7 & 11 & 11 & -103 & $i$ & 4 & & -9 & 26 & & & & \\
\hline-2 & 16 & -29 & 5 & & 46 & & 60 & & -8 & & & & & \\
\hline-1 & 162 & 16 & 8 & & & 20 & & & & 33 & & & & 0 \\
\hline 0 & 11 & 11 &,$L=$ & & 11 & 21 & 4 & -43 & $x-6$ & & -8 & 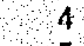 & & \\
\hline 1 & 2 & -2 & & 19 & -22 & 22 & 23 & 27 & -5 & 268 & & & 37 & 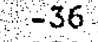 \\
\hline 2 & 48 & 4 & & & 30 & $K, L=$ & $=$ & & -4 & 376 & & & 117 & 13 \\
\hline 3 & 5 & -5 & & & -14 & & 30 & -29 & -3 & 18 & -18 & 7 & 50 & \\
\hline 4 & 87 & & -13 & & -38 & -21 & & 23 & -2 & & & & 175 & 17 \\
\hline 5 & 2 & 2 & & & 40 & -28 & 8 & -85 & -1 & 229 & & 9 & 41 & \\
\hline 6 & 5 & -6 & & & 4 & -19 & 7 & -79 & & & & 10 & 6 & \\
\hline 10 & 6 & 5 & & & 5 & -17 & & -62 & 1. & 107 & & 12 & 106 & -108 \\
\hline 12 & 2 & -2 & & & 2 & -16 & & & & & & 14 & & \\
\hline 1 & 14 & -2 & -8 & 9 & 8 & 15 & 165 & -167 & 4 & & & 17 & & \\
\hline $\mathrm{L}=$ & 1 & 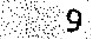 & - & 6 & 5 & -13 & 66 & 57 & 5 & 12 & 119 & 19 & 28 & -25 \\
\hline & 61 & 6 & -4 & 6 & & & & 100 & & 9 & -98 & $\mathrm{~K} ; \mathrm{T}_{1}=$ & & \\
\hline 2 & 32 & -3 & -3 & & & 0 & 64 & & & 45 & 45 & -21 & 35 & -3 \\
\hline & 28 & & - & & & & & & & 338 & & -18 & 99 & \\
\hline & 44 & 4 & -1 & 6 & 5 & -8 & 2 & & & & & -17 & & \\
\hline & 44 & 4 & 0 & & & -7 & & & & 1 & -1 & -16 & 19 & -9 \\
\hline 1 & 52 & -4 & 1 & 3 & -31 & -6 & & & & & & & & \\
\hline & 13 & 14 & 2 & & -3 & -5 & & & & 5 & & & 9 & -7 \\
\hline-5 & 8 & 9 & 5 & & 286 & -4 & 2 & & 4 & 109 & & -13 & 42. & -4 \\
\hline-8 & 32 & -3 &,$L=$ & 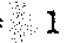 & 12 & -3 & & & & & & -1 & 3 & \\
\hline-7 & & -12 & & 27 & & & & & & & & -11 & 189 & -15 \\
\hline & & & & & & -1 & 13 & & & & & -9 & & \\
\hline-5 & 16 & 16 & -10 & & -4 & 0 & 7 & & & & - & 8 & & \\
\hline-4 & & 4 & -9 & & & 1 & & & & & & -7 & 00 & -2 \\
\hline-3 & 17 & 17 & $-\varepsilon$ & & & 2 & & & $K, L=$ & & & -6 & 70 & -17 \\
\hline-2 & 7 & 6 & -7 & & $-\varepsilon$ & & & & & & & & & \\
\hline-1 & 71 & 7 & -4 & & -2 & & & & -22 & & & -4 & & \\
\hline 0 & 6 & $\Leftrightarrow$ & -1 & & & 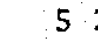 & & & & & & & & \\
\hline 2 & & 3 & & & & & & & & & & -2 & 204 & -2 \\
\hline 3 & 4 & -4 &,$L=$ & & & 7 & & & & & & -1 & 6 & \\
\hline 7 & 4 & 4 & -8 & 4 & & 8 & & & & & & & & \\
\hline 8 & & 2 & -7 & & & 9 & & $?$ & -17 & & & 1 & 20 & -2 \\
\hline 10 & & 3 & -6 & & -2 & 10 & & & -16 & & & & 0 & \\
\hline$L=$ & 1 & 1 & -4 & & -3 & 11 & 152 & -126 & -15 & & & & 389 & \\
\hline & 31 & & $K, L=$ & & & 12 & 5 & & & & & & 172 & \\
\hline & 40 & -3 & 0 & 56 & -5 & 16 & 3 & -2 & -13 & & & & 127 & -12 \\
\hline & 35 & 3 & 1 & & & & 3 & -3 & -12 & & & & & \\
\hline & 40 & & & & & 1 & & & -1 & & & & 185 & 19 \\
\hline & & 5 & & & & 20 & 2 & & & & & 8 & 72 & \\
\hline & & -3 & 5 & & & 21 & 48 & -53 & -8 & 188 & -2 & & & \\
\hline & & 4 & & & & $K, L=$ & $=$ & & & & & 0 & & -2 \\
\hline-5 & & 10 & 8 & & & & 96 & -9 & -6 & & & 11 & 124 & 13 \\
\hline-8 & 01 & 23 & 93 & & -131 & -19 & 68 & -71 & -5 & & & & & \\
\hline & & 171 & 111 & & 90 & -17 & 33 & -22 & -4 & 250 & 242 & 13 & 25 & 2. \\
\hline
\end{tabular}




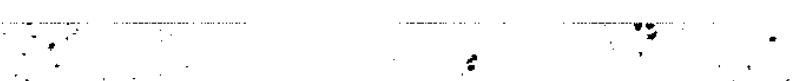

\section{(4)}

\begin{tabular}{|c|c|c|c|c|c|c|c|c|c|c|c|c|c|c|}
\hline 11 & & $\mathrm{FC}$ & $\mathrm{H}$ & $\mathrm{FO}$ & $\mathrm{FC}$ & 11 & EO & $\mathrm{FC}$ & $\mathrm{H}$ & Fo & $\begin{array}{l}\mathrm{FC} \\
39\end{array}$ & $\begin{array}{l}11 \\
20\end{array}$ & 24 & -21 \\
\hline 15 & 102 & -96 & -5 & 23 & 26 & $\begin{array}{r}-17 \\
-16\end{array}$ & 21 & 118 & $\begin{array}{l}-14 \\
-12\end{array}$ & $\begin{array}{l}45 \\
42\end{array}$ & $\begin{array}{r}39 \\
-36\end{array}$ & $\begin{array}{l}20 \\
21\end{array}$ & 30 & $\begin{array}{l}-21 \\
-31\end{array}$ \\
\hline 15 & $\begin{array}{l}23 \\
285\end{array}$ & $\begin{array}{r}-21 \\
-24\end{array}$ & $\begin{array}{l}-4 \\
-3\end{array}$ & 18 & 21 & $\begin{array}{l}-16 \\
-15\end{array}$ & 69 & $\begin{array}{l}-63 \\
-64\end{array}$ & $\begin{array}{l}-12 \\
-11\end{array}$ & 44 & 46 & $\mathrm{~K}, \mathrm{~L}=$ & & \\
\hline 17 & 57 & -59 & $\begin{array}{l}-3 \\
-2\end{array}$ & 18 & -105 & -13 & 49 & 49 & -10 & 90 & 91 & -21 & 27 & -19 \\
\hline$K, L=$ & 2 & 5 & -1 & 00 & -100 & -12 & 30 & -141 & $-7 I$ & 32 & 146 & -29 & 24 & 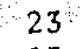 \\
\hline & 21 & -21 & 1 & 45 & 51 & -10 & 30 & -143 & -4 & 45 & -30 & -19 & r & -85 \\
\hline & 71 & 76 & 2 & 29 & -23 & -9 & 105 & -115 & -3 & 32 & 37 & -18 & & -26 \\
\hline & 29 & -29 & 3 & 21 & -127 & -8 & 46 & -39 & -2 & 66 & -67 & -15 & & -47 \\
\hline & 161 & -167 & 5 & 29 & 245 & -7 & 29 & -153 & 0 & 68 & 50 & $-14^{\circ}$ & & 11. \\
\hline 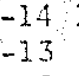 & 127 & 128 & 6 & 40 & -35 & -6 & & -69 & 1 & 44 & -40 & -13 & & 142 \\
\hline 2 & $\begin{array}{r}52 \\
249\end{array}$ & -5 & 7 & 92 & -98 & -5 & 46 & & 3 & 46 & -43 & -12 & & 8 \\
\hline 11 & $\begin{array}{r}249 \\
51\end{array}$ & 277 & 8 & 36 & -3 & -41 & 121 & 142 & 4 & 104 & 107 & $=0$ & & 10 \\
\hline 10 & $\begin{array}{l}51 \\
96\end{array}$ & $\begin{array}{r}-47 \\
08\end{array}$ & 9 & 28 & -26 & -3 & 90 & -105 & 5 & 25 & 12 & $=?$ & & \\
\hline-9 & $\begin{array}{l}96 \\
82\end{array}$ & -98 & 12. & 46 & 39 & -2 & 80 & -87 & 6 & 38 & 4. & -8 & & -2 \\
\hline-8 & $\begin{array}{r}82 \\
183\end{array}$ & $\begin{array}{r}85 \\
166\end{array}$ & 16 & 18 & -18 & -11 & 170 & 19 & 7 & 30 & 32 & -7 & & -93 \\
\hline-7 & 71 & $\begin{array}{r}166 \\
-62\end{array}$ & $\mathrm{~K}, \mathrm{~L}=$ & 2 & 7 & 0 & & & 8 & 28 & 35 & -6 & & 122 \\
\hline-5 & 177 & 17 & -22 & 25 & 34 & 1 & 70 & -71 & $K, L=$ & & 11 & -5 & & \\
\hline-4 & 95 & 17 & $\begin{array}{l}-21 \\
-20\end{array}$ & 54 & -54 & 2 & 10 & -10 & $\begin{array}{l}-16 \\
-13\end{array}$ & 30 & -28 & -4 & 24 & $\begin{array}{l}-24 \\
-30\end{array}$ \\
\hline-3 & 44 & -4 & $\begin{array}{l}-20 \\
-19\end{array}$ & 5 & -22 & 3 & 20 & -3 & & 55 & 53 & -3 & 9 & $\begin{array}{l}30 \\
-5\end{array}$ \\
\hline-2 & 249 & -241 & 19 & 89 & 88 & $\begin{array}{l}4 \\
5\end{array}$ & $\begin{array}{l}55 \\
32\end{array}$ & -3 & $\begin{array}{l}-11 \\
-10\end{array}$ & 43 & -38 & -2 & 3 & $\begin{array}{l}-5 \\
12\end{array}$ \\
\hline-1 & 255 & -25 & $\begin{array}{l}-17 \\
-16\end{array}$ & $\begin{array}{l}91 \\
85\end{array}$ & $\begin{array}{l}-8 \\
-9\end{array}$ & $\epsilon$ & 75 & -70 & $\begin{array}{r}-10 \\
-9\end{array}$ & 60 & 60 & $\begin{array}{r}-1 \\
0\end{array}$ & 31 & $\begin{array}{l}12 \\
-2\end{array}$ \\
\hline 0 & 196 & 21 & -14 & $\begin{array}{r}85 \\
122\end{array}$ & $\begin{array}{r}-93 \\
131\end{array}$ & 7 & 4 & -4 & $\begin{array}{l}-9 \\
-8\end{array}$ & $\begin{array}{l}46 \\
62\end{array}$ & 43 & $\begin{array}{l}0 \\
1\end{array}$ & 72 & $\begin{array}{l}-29 \\
-61\end{array}$ \\
\hline 1 & 248 & $-24 !$ & -13 & 51 & 58 & 9 & 19 & -1 & $\begin{array}{l}-8 \\
-7\end{array}$ & 62 & 53 & $\begin{array}{l}1 \\
2\end{array}$ & & -61 \\
\hline 2 & 78 & 8 & -12 & $7 \epsilon$ & -77 & 11 & 2. & 1 & -6 & $\begin{array}{l}79 \\
47\end{array}$ & 87 & 2 & & \\
\hline 3 & 28 & 28 & -11 & 96 & 9 & 12 & 48 & 4 & 4 & $\begin{array}{l}47 \\
67\end{array}$ & -4 & $\begin{array}{l}3 \\
4\end{array}$ & & -8 \\
\hline 4 & 111 & 11 & -10 & 54 & -59 & $\mathrm{~K}, \mathrm{~L}=$ & 6 & & 5 & $\begin{array}{l}61 \\
38\end{array}$ & -3 & 5 & 267 & 3 \\
\hline 5 & 141 & -14 & -9 & 64 & -65 & -19 & 29 & 19 & $K, L=$ & 2 & 1 & 6 & & -5 \\
\hline 6 & 42 & -4 & -8 & 48 & 42 & -18 & 39 & 3 & -11 & 45 & -3 & 7 & & -5 \\
\hline 8 & 148 & 143 & -7 & 93 & -94 & -14 & 30 & $2^{\circ}$ & -10 & & 2 & 11 & & 3 \\
\hline 9 & 70 & 4 & -6 & 32 & 36 & -13 & 32 & & -9 & 31 & $2^{\circ}$ & 12 & 41 & -3 \\
\hline 10 & 152 & -156 & $\imath-5$ & 78 & 94 & -121 & 126 & -12 & -6 & 23 & 2 & 13 & 4 & -3 \\
\hline 12 & 47 & -4 & -3 & 32 & -3 & -10 & 33 & -5 & -5 & 60 & -5 & $14:$ & & \\
\hline 13 & 38 & -3 & -2 & 71 & $-7 c$ & -9 & 128 & -14 & -2 & 3 & 3 & 15 & & \\
\hline 15 & 35 & -3 & -1 & 4 & -4 & -7 & 5 & & -1 & 4 & 3 & 17 & & \\
\hline 16 & 18 & -2 & 0 & & 13 & -5 & 7 & 7 & 1 & 35 & -3 & 19 & 3 & \\
\hline 17 & 14. & 15 & 1 & & 19 & -4 & 8 & -8 & $\mathrm{~K}, \mathrm{~L}=$ & & 0 & 20 & & \\
\hline$k, k=$ & 2 & 6 & 2 & 21 & -24 & -3 & 5 & -6 & 1 & 7 & .72 & $K, L=$ & & \\
\hline-20 & 69 & -62 & 3 & 17 & 17 & -2 & 5 & 5 & 2 & & -7 & -20 & & -0 \\
\hline-18 & 93 & 7 & 4 & 12 & -12 & 0 & 3 & 2 & 3 & 205 & -18 & -1 & & \\
\hline-17 & 91 & -9 & 5 & 7 & -6 & 1 & 49 & 4 & 4 & 32 & 28 & -1 & & -5 \\
\hline-16 & 44. & -5 & 6 & 8 & -8 & 2 & 5 & -4 & 5 & 179 & -14 & -16 & & \\
\hline-15 & 28 & 2 & 7 & 2 & 1 & 3 & 6 & -5 & 61 & 188 & -1 & -1 & & \\
\hline-1 & 162 & 18 & 9 & 2 & -1 & 4 & 87 & & 7 & 4 & -3 & -13 & & \\
\hline-13 & 242 & -26 & 10 & 5 & -5 & 5 & 4 & -6 & 10 & & & -11 & & -1 \\
\hline-12 & 157 & 16 & 12 & 5 & 5 & 6 & 4 & & 11 & 113 & -1 & 10 & & \\
\hline-11 & 232 & 25 & 13 & 5 & -5 & 8 & & & 12 & & -10 & -9 & & 10 \\
\hline-10 & 182 & 19 & 14 & 20 & 18 & 9 & 87 & & 131 & 10 & 10 & - & & \\
\hline-9 & 80 & 8 & $K, L=$ & 2 & 8 & $K, L=$ & 2 & & $1:$ & & -9 & -7 & & \\
\hline-7 & 24 & -23 & -20 & 73 & 6 & -18 & 15 & -1 & 16 & & -4 & -6 & 16 & \\
\hline-6 & 42 & 39 & -19 & 54 & -50 & -17 & 31 & -29 & 17 & 29 & -28 & -5 & 79 & \\
\hline
\end{tabular}


(5)

\begin{tabular}{|c|c|c|c|c|c|c|c|c|c|c|c|c|c|}
\hline & & 4 & Fo & $\mathrm{FC}$ & 11 & 1.0 & I. & 4 & & & $\mathrm{H}$ & & \\
\hline 118 & -106 & 211 & 108 & 138 ; & 52 & 219 & 240 & -10 & 92 & 107 & -14 & 42 & 37 \\
\hline 75 & 72 & -19 & 35 & -328 & 62 & 242 & 272 & 49 & 69 & 73 & -8 & & \\
\hline 144 & 156 & -16 & 42 & 36 & 7 & 117 & -125 & $-8 \quad 6$ & 68 & -73 & -7 & 50 & \\
\hline 86 & $\leqslant 1$ & -15 & 48 & -46 & 8 & 45 & -53 & -714 & 44 & -171 & -6 & & 87 \\
\hline 215 & -237 & -141 & 103 & -99 & +9 & 82 & 85 & -6 & 23 & -26 & -5 & & \\
\hline 212 & 223 & -13 & 30 & 31 & 10 & 36 & 39 & -510 & .04 & 119 & -4 & & -40 \\
\hline 37 & 33 & -121 & 143 & -140 & 11 & 35 & -41 & -4 & 22 & 24 & 3 & & \\
\hline 77 & -77 & -112 & 295 & -325 & 12 & 32 & 31 & -3 & 35 & 32 & -2 & & \\
\hline 177 & -158 & -10 & 41 & -36 & 14 & 41 & -48 & -2 & 80 & -79 & -1 & & \\
\hline & 84 & -9 & 134 & 146 & 15 & 75 & -75 & -1 & 63 & 62 & 0 & & -65 \\
\hline 111 & -118 & -8 & 41 & -44 & 16 & 19 & -20 & 0 & 88 & 9 & 3 & & \\
\hline 92 & 93 & -7 & 95 & -105 & $k, L=$ & 3 & 6 & 11 & 73 & -19 & 4 & & -69 \\
\hline $\begin{array}{l}60 \\
58\end{array}$ & -58 & -6 & 61 & -57 & -21 & 46 & -42 & 2 & 72 & -8 & 6 & & -44 \\
\hline $\begin{array}{l}58 \\
97\end{array}$ & -49 & -5 & 47 & 46 & -20 & 29 & -23 & 3 & & 8 & 21 & & \\
\hline $\begin{array}{r}97 \\
100\end{array}$ & 111 & -41 & 178 & 179 & -19 & 80 & -72 & 41 & 107 & 124 & $\mathrm{~K}, \mathrm{~L}=$ & 3 & \\
\hline $\begin{array}{r}100 \\
38\end{array}$ & 117 & -3 & 140 & -158 & -18 & .46 & 47 & 51 & & -134 & -17 & 17 & \\
\hline $\begin{array}{l}38 \\
25\end{array}$ & -31 & -1 & 89 & 91 & -16 & 100 & -9 & 6 & 83 & -91 & $-16-$ & & \\
\hline $\begin{array}{r}25 \\
104\end{array}$ & 18 & 1 & 46 & -44 & -15 & 159 & 15 & 7 & 66 & -109 & -15 & 4 & -1 \\
\hline $\begin{array}{r}104 \\
34\end{array}$ & 106 & 4 & 63 & 72 & -13 & 96 & 106 & 8 & 57 & -67 & -14 & 25 & -2 \\
\hline $\begin{array}{l}34 \\
3\end{array}$ & 38 & 5 & 48 & -48 & -12 & 34 & & 10 & 20 & -21 & -13 & 3 & \\
\hline $\begin{array}{r}3 \\
67\end{array}$ & 3 & 6 & 50 & 47 & -11 & 56 & 5 & 11 & 42 & 45 & -10 & & 10 \\
\hline $\begin{array}{l}67 \\
66\end{array}$ & 65 & 7 & 54 & 53 & -10 & 147 & 153 & 13 & & 2 & -9 & 72 & \\
\hline $\begin{array}{l}66 \\
34\end{array}$ & -65 & 8 & 25 & 18 & -9 & 209 & & $K, L=$ & & & -7 & 53 & \\
\hline $\begin{array}{l}34 \\
32\end{array}$ & 34 & 10 & 81 & 84 & -8 & 28 & -28 & .20 & & 5 & -6 & 80 & \\
\hline $\begin{array}{l}32 \\
78\end{array}$ & 30 & 11 & 82 & $-8 c$ & -7 & 65 & -67 & -17 & & 43 & -5 & 53 & \\
\hline $\begin{array}{l}78 \\
74 .\end{array}$ & -78 & 12 & 35 & 3 & -6 & 58. & 56 & -16 & 46 & 4 & -4 & 77 & \\
\hline $\begin{array}{l}74 \\
81\end{array}$ & -70 & 13 & 23 & -1 & -4 & $4 ?$ & 44 & -15 & 73 & -6 & -31 & 103 & \\
\hline $\begin{array}{r}81 \\
218\end{array}$ & -84 & 14 & 55 &.-49 & -3 & 62 & -60 & -14 & 63 & 60 & -1 & -3 & \\
\hline $\begin{array}{ll}11 & 218 \\
10 & 209\end{array}$ & 208 & 15 & 46 & -48 & -2 & 46 & -44 & -131 & 131 & -12 & 2 & 39 & -40 \\
\hline $\begin{array}{rr}10 & 209 \\
-9 & 47\end{array}$ & -212 & 16 & 17 & 16 & 0 & $54^{r}$ & -54 & -12 & 87 & 78 & 3 & & 4 \\
\hline $\begin{array}{l}47 \\
45\end{array}$ & 46 & $K, L=$ & 3 & & 1 & 40 & 45 & -111 & 111 & -13 & 5 & 35 & \\
\hline 45 & 47 & -19 & 59 & -50 & 2 & 106 & 113 & -8 & 95 & -11 & 6 & 26 & \\
\hline 152 & -14 & -18 & 41 & 35 & 3 & 22 & -2 & -7 & 82 & -88 & $K, L=$ & & \\
\hline 150 & 15 & -17 & 75 & 7 & 4 & 87 & 92 & -6 & 67 & 7 & -14 & 43 & \\
\hline 54 & -6 & -16 & 68 & -5 & 6 & 36 & -3 & -5 & 8 & & -13 & 85 & \\
\hline 107 & 9 & -15 & 47 & 42 & 7 & 57 & 6 & -2 & 52 & -5 & -12 & 23 & \\
\hline 126 & 13 & -13 & 56 & 5 & 8 & 23 & & -1 & 3 & & -10 & 64 & \\
\hline 264 & 278 & -12 ? & 209 & -22 & 12 & 31 & -3 & 0 & 37 & & -9. & 2 & \\
\hline 29 & 1 & -11 & 129 & 13 & 13 & 32 & 3 & 2 & 33 & & -7 & & \\
\hline 1181 & -18 & -10 & 194 & 21 & 14 & 5 & -5 & 3 & 91 & -10 & -4 & & \\
\hline 2131 & -13 & & 13 & 13 & 15 & 17 & 2 & 4 & 66 & -7 & -3 & & \\
\hline 40 & -4 & -7 & 83 & -8 & $\mathrm{~K}, \mathrm{~L}=$ & & & 5 & 2 & -2 & -2 & 36 & \\
\hline $\begin{array}{ll}5 & 67\end{array}$ & 87 & -5 & 120 & -12 & & 20 & 1 & 6 & 69 & -7 & 3 & 2 & \\
\hline 6100 & -112 & $\triangle A$ & 81 & 88 & -19 & 70 & -6 & 7 & 3 & -3 & $K, L=$ & & \\
\hline $\begin{array}{l}7 \\
72\end{array}$ & -10 & -3 & 30 & -3 & -18 & 54 & -5 & 8 & 36 & -3 & -8 & & \\
\hline 8126 & 13 & -2 & 7 & 7 & -17 & 11 & 10 & 10 & 3. & & -6 & 41 & \\
\hline $9 \quad 32$ & -2 & -1 & 4 & -3 & -16 & 104 & & 1.1 & 23 & -24 & & & \\
\hline 10134 & -14 & 0 & -8 & -7 & -15 & 158 & -15 & $K, L=$ & 3 & & 8.4 & & \\
\hline 1151 & 53 & 1 & & & -14 & 79 & -7 & -18 & 77 & & -3 & 33 & \\
\hline $12 \quad 102$ & 115 & 2 & 9 & 10 & -13 & & & -17 & 2 & & $K, L=$ & & \\
\hline$G, Y=3$ & & 3 & 85 & & -12 & 110 & 13 & -16 & 26 & & 0 & & \\
\hline 31 & -40 & 4 & 29 & -32 & -11 & 72 & -72 & -15 & 66 & -55 & 1 & 70 & \\
\hline
\end{tabular}




\begin{tabular}{|c|c|c|c|c|c|c|c|c|c|c|c|c|c|c|}
\hline $\mathrm{H}$ & 10 & $F C$ & 413 & $\mathrm{FO}$ & $\mathrm{FC}$ & $\mathrm{H}$ & $\mathrm{FO}$ & FC & $\mathrm{H}$ & ro & $\mathrm{FC}$ & $\mathrm{H}$ & FO & FC \\
\hline 2 & 62 & 62 & -13 & 77 & 78 & 4 ? & 97 & -115 & 7 & 66 & 72 & 14 & 38 & -37 \\
\hline & 357 & 325 & -12 & 97 & 110 & & 121 & -132 & -6 & 26 & -23 & -13 & 29 & $\begin{array}{l}-27 \\
-51\end{array}$ \\
\hline $\begin{array}{l}4 \\
51\end{array}$ & 98 & 90 & -11 & 56 & 52 & 61 & 109 & -119 & -41 & 112 & -126 & -12 & 49 & $\begin{array}{r}-51 \\
36\end{array}$ \\
\hline $\begin{array}{l}5 \\
7\end{array}$ & 165 & -154 & -10 & 29 & -17 & 7 & 89 & 97 & -2 & 64 & -74 & -11 & 31 & $\begin{array}{r}36 \\
-27\end{array}$ \\
\hline & 121 & 114 & -92 & $250^{\circ}$ & 280 & 8 , & 29 & 31 & -1 & 81 & 79 & -10 & $\begin{array}{l}26 \\
38\end{array}$ & $\begin{array}{r}-27 \\
34\end{array}$ \\
\hline 11 & 45 & 44 & -8 & 75 & $\begin{array}{r}77 \\
219\end{array}$ & $\begin{array}{r}9 \\
11\end{array}$ & 163 & -171 & $\begin{array}{lll}0 & 1\end{array}$ & 113 & $\begin{array}{r}122 \\
25\end{array}$ & $\begin{array}{l}-9 \\
-8\end{array}$ & $\begin{array}{l}38 \\
38\end{array}$ & 42 \\
\hline $\begin{array}{l}11 \\
13\end{array}$ & 16 & -116 & -7 & 205 & $\begin{array}{r}219 \\
-111\end{array}$ & $\begin{array}{l}111 \\
14\end{array}$ & 107 & 115 & 2 & $\begin{array}{l}24 \\
75\end{array}$ & $\begin{array}{r}25 \\
-79\end{array}$ & -7 & 50 & -57 \\
\hline $\begin{array}{l}13 \\
14\end{array}$ & 141 & $\begin{array}{r}-141 \\
-37\end{array}$ & $\begin{array}{l}-6 \\
-5\end{array}$ & 100 & $\begin{array}{r}-111 \\
-15\end{array}$ & $\begin{array}{l}14 \\
15\end{array}$ & 38 & $\begin{array}{r}40 \\
-45\end{array}$ & $\frac{2}{3}$ & $\begin{array}{l}75 \\
45\end{array}$ & 43 & -5 & 26 & -28 \\
\hline 15 & 73 & $\begin{array}{r}-37 \\
73\end{array}$ & $\begin{array}{l}-5 \\
-4\end{array}$ & $\begin{array}{r}24 \\
155\end{array}$ & $\begin{array}{r}-15 \\
170\end{array}$ & 816 & 41 & 43 & 4 & $\begin{array}{l}42 \\
66\end{array}$ & 75 & -3 & 44 & -42 \\
\hline $\begin{array}{l}16 \\
17\end{array}$ & 31 & -81 & -3 & 101 & -96 & $\mathrm{R}, \mathrm{L}=$ & 4 & 4 & 52 & 205 & 202 & -2 & 87 & 93 \\
\hline 17 & 30 & -24 & -2 & 162 & -178 & -20 & 31 & 32 & 6 & 33 & 34 & 0 & 44 & 44 \\
\hline $\begin{array}{l}18 \\
19\end{array}$ & 70 & 65 & -1 & 78 & 72 & -19 & 43 & 49 & 7 & 38 & 34 & 2 & 44 & -45 \\
\hline $\begin{array}{l}19 \\
K, L=\end{array}$ & 22 & -24 & 0 & 90 & 88 & -18 & 35 & 37 & 8 & 80 & 78 & 3 & 37 & -35 \\
\hline $\begin{array}{l}K, L= \\
-18\end{array}$ & 4 & 1 & 1 & 37 & 37 & -17 & 51 & -53 & 10 & 42 & -41 & 4 & 40 & 36 \\
\hline $\begin{array}{l}-18 \\
-17\end{array}$ & 32 & 35 & 2 & 66 & -68 & -16 & 46 & -54 & 11 & 27 & 29 & 6 & 29 & -26 \\
\hline $\begin{array}{l}-17 \\
-16\end{array}$ & 70 & 73 & 3 & 135 & -148 & -15 & 48 & -45 & 12 & 21 & -18 & 7 & 75 & -74 \\
\hline $\begin{array}{l}-16 \\
-14\end{array}$ & 37 & -30 & 4 & 77 & 71 & -10 & 40 & 33 & 13 & 38 & -36 & 8 & 35 & 29 \\
\hline $\begin{array}{l}-14 \\
-13\end{array}$ & 71 & 67 & 5 & 26 & -231 & -13 & 71 & 87 & 14 & 22 & 21 & 9 & 22 & -27 \\
\hline $\begin{array}{l}-131 \\
-121\end{array}$ & 165 & 157 & 6 & 86 & -94 & -121 & 124 & -123 & $K, L=$ & 44 & 6 & 10 & 19 & -11 \\
\hline $\begin{array}{l}-12 \\
-11\end{array}$ & 113 & 104 & 8 & 41 & 41 & -11 & 205 & -235 & -18 & 59 & -61 & 11 & 20. & 21 \\
\hline $\begin{array}{l}-11 \\
-10\end{array}$ & 124 & 138 & 9 & 72 & 86 & -9 & 38 & 37 & -17 & 61 & -60 & $K, L=$ & 4 & 8 \\
\hline $\begin{array}{r}-10 \\
-8\end{array}$ & 44 & 43 & 10 & 79 & -79 & -8 & 44 & -47 & -16 & 25 & 23 & -16 & .26 & -28 \\
\hline $\begin{array}{l}-8 \\
-7\end{array}$ & 34 & 26 & 11 & 86 & -78 & -7 & 118 & 135 & -15 & 86 & 88 & -15 & 52 & 48 \\
\hline $\begin{array}{l}-7 \\
-6\end{array}$ & 29 & 8 & 12 & 54 & 44 & -5 & 60 & -63 & -14 & 28 & -27 & -12 & 56 & -54 \\
\hline $\begin{array}{l}-6 \\
-5\end{array}$ & 43 & -38 & 13 & 130 & 136 & -4 & 23 & -16 & -13 & 25 & -30 & -11 & 82 & 80 \\
\hline $\begin{array}{l}-5 \\
-4\end{array}$ & 65 & -67 & 14 & 77 & -72 & -3 & 37 & 37 & -11 & 32 & -22 & -10 & .74 & 72 \\
\hline $\begin{array}{l}-4 \\
-3\end{array}$ & 43 & 39 & 15 & 72 & 78 & -2 & 39 & -35 & -10 & 30 & 15 & -8 & 60 & $\begin{array}{r}-56 \\
50\end{array}$ \\
\hline $\begin{array}{l}-3 \\
-2\end{array}$ & 66 & -45 & 16 & 34 & 40 & -1 & 49 & 51 & -91 & 102 & 115 & -6 & 49 & \\
\hline $\begin{array}{l}-2 \\
-1\end{array}$ & 133 & -124 & 17 & 42 & -44 & 2 & 80 & -87 & -8 & 26 & 30 & -5 & 21 & $\begin{array}{r}-22 \\
77\end{array}$ \\
\hline $\begin{array}{r}-1 \\
0\end{array}$ & 25 & 14 & 18 & 20 & -21 & 4 & 52 & -49 & -7 & 54 & -64 & -4 & 83 & $\begin{array}{l}77 \\
48\end{array}$ \\
\hline $\begin{array}{l}0 \\
1\end{array}$ & 93 & 95 & $K_{2} L=$ & 4 & 3 & 71 & 176 & 182 & -61 & 119 & -135 & -3 & 50 & $\begin{array}{l}48 \\
73\end{array}$ \\
\hline $\begin{array}{l}1 \\
2\end{array}$ & 85 & 82 & -20 & 56 & 66 & 8 & 45 & -48 & -4 & 41 & 48 & -2 & 82 & 73 \\
\hline 2 & 57. & 52 & -17 & 59 & -69 & 9 & 38 & -37 & -3 & 45 & -48 & 0 & 45 & -40 \\
\hline 3 & 66 & 71 & -15 & 45 & -43 & 10 & 23 & 17 & -2 & 41 & -47 & 1 & 64 & -58 \\
\hline 4 & 141 & 132 & -14 & 49 & 44 & 11 & 51 & -43 & -1 & 41 & 42 & 3 & 5.6 & 49 \\
\hline 5 & 77 & -74 & -13 & 67 & -66 & 13 & 45 & 44 & 0 & 81 & 80 & 4 & 43 & 35 \\
\hline 7 & 57 & 60 & -12 & 35 & -24 & 14 & 38 & 33 & 1 & 22 & -29 & 5 & 55 & -53 \\
\hline 8 & 48 & 54 & -11 & 146 & -166 & 15 & 69 & -78 & 2 & 79 & -80 & 6 & 37 & -38 \\
\hline 9 & 30 & 24 & -10 & 106 & -116 & 16 & 9 & -8 & 3 & 26 & 26 & 7 & 57 & -50 \\
\hline 10 & 43 & -47 & -8 & 48 & 148 & $\mathrm{~K}, \mathrm{~L}=$ & 4 & & 41 & 156 & 269 & 8 & 38 & -32 \\
\hline 11 & 90 & -89 & -7 & $\$ 2$ & -85 & -20 & 32 & -37 & 5 & 73 & 71 & 9 & 27 & 28 \\
\hline 12 & 71 & -65 & -6 & 38 & 40 & -19 & 48 & 47 & 8 & 28 & 28 & $K, L=$. & 4 & $\begin{array}{r}9 \\
\end{array}$ \\
\hline 13 & 223 & 207 & -5 & 89 & 96 & -18 & 49 & -45 & 9 & 76 & -72 & -16 & 20 & -16 \\
\hline 14 & 110 & -98 & -4 & 58 & 57 & -17 & 25 & -22 & 10 & 24 & -21 & -15 & 25 & 22 \\
\hline 17 & 25 & 29 & -3 & 57 & -56 & -15 & 69 & 71 & 11 & 39 & 37 & -14 & 33 & 34 \\
\hline $\mathrm{K}, \mathrm{L}=$ & $=4$ & 2 & -2 & 115 & -128 & -14 & 78 & 73 & 12 & 69 & 64 & -13 & 21 & -21 \\
\hline-20 & 18 & 18 & -1 & 56 & 55 & -13 & 44 & -48 & 13 & 33 & -35 & -12 & 23 & 12 \\
\hline-19 & 26 & 28 & 0 & 113 & 113 & -12 & 65 & -65 & $\mathrm{~K}, \mathrm{~L}=$ & 4 & 7 & -11 & 40 & 39 \\
\hline-38 & 31 & 32 & 1 & 60 & -57 & -11 & 55 & 52 & -17 & 43 & -43 & -10 & 45 & 43 \\
\hline-17 & 41 & -51 & 2 & 124 & -129 & -9 & 27 & 27 & -16 & 20 & 23 & -9 & 25 & $\begin{array}{r}-29 \\
38\end{array}$ \\
\hline-14 & 73 & 72 & 3 & 176 & -184 & -8 & 33 & 27 & -15 & 51 & 52 & -8 & 43 & \\
\hline
\end{tabular}




\section{:}

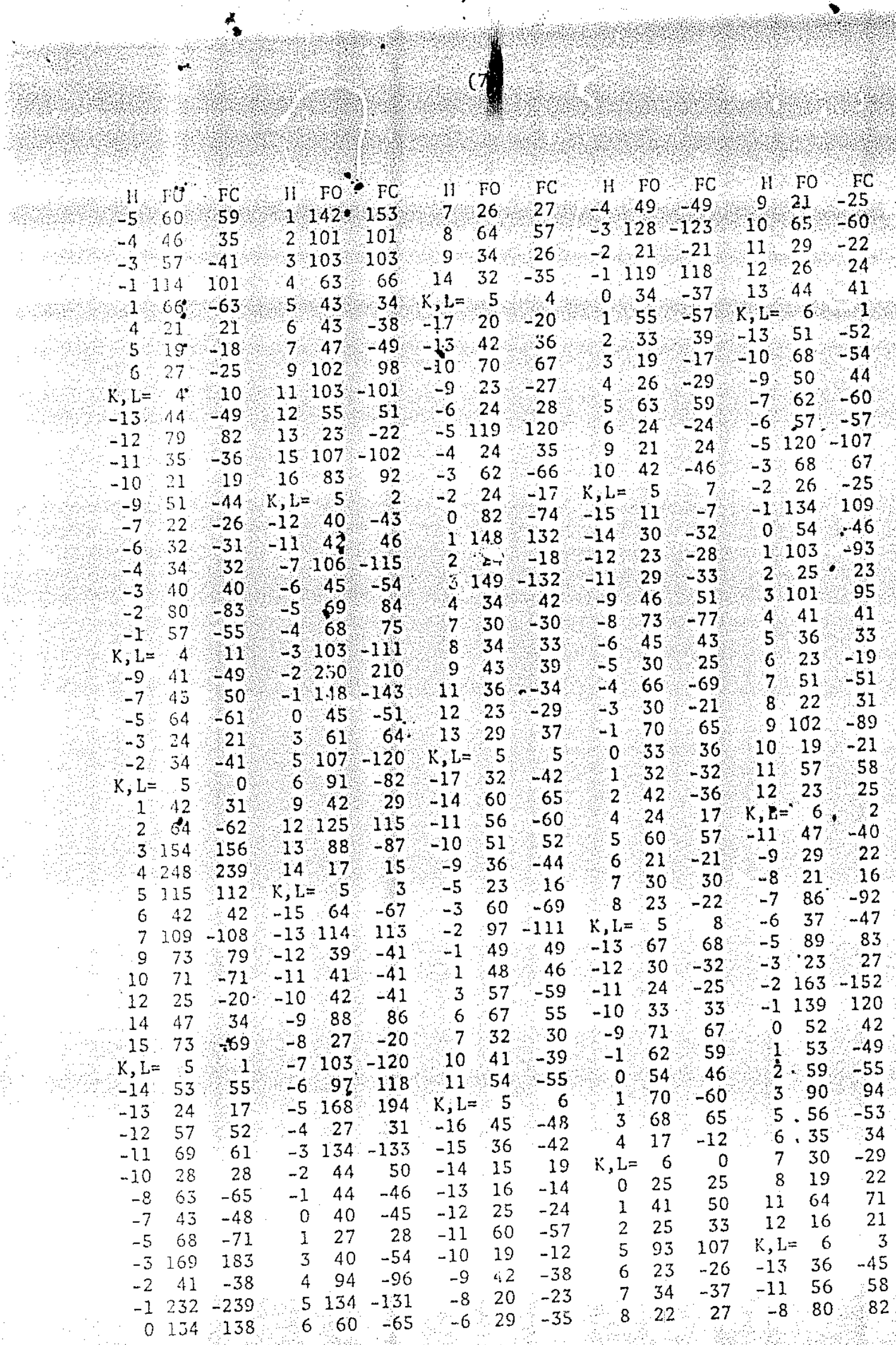


(8)

\begin{tabular}{|c|c|c|c|c|c|c|c|c|c|c|c|c|c|c|}
\hline 11 & $\mathrm{FO}$ & FC & H & $\mathrm{FO}$ & $\mathrm{FC}$ & $H$ & $\mathrm{I} Q \mathrm{Q}$ & $F C$ & $\mathrm{H}$ & Fo & $\mathrm{FC}$ & $\mathrm{H}$ & FO & FC \\
\hline-6 & 87 & -83 & 0 & 114 & 110 & 6 & 13 & -19 & -1 & 32 & 29 & -4 & 39 & 44 \\
\hline-5 & 36 & 51 & 3 & 18 & 20 & $k, l=$ & $=6$ & 06 & 0 & 50 & -46 & -2 & 40 & -32 \\
\hline-4 & 88 & 91 & 4 & 24 & 18. & -10 & 26 & -26 & 1 & 38 & 43 & 0 & 22 & 21 \\
\hline-3 & 1.18 & 121 & 5 & 43 & -40 & -9 & 12 & -13 & 2 & 21 & -26 & 2 & 15 & -14 \\
\hline-2 & 59 & -64 & 7 & 23 & 20 & -8 & 47 & 42 & $K, L=$ & 7 & 0 & 5 & 41 & 39 \\
\hline-1 & 34 & -34 & 8 & 19 & -19 & -6 & 14 & -12 & 3 & 45 & -36 & $K, L=$ & 7 & 3 \\
\hline 0 & 34 & 32 & 9 & 34 & 37 & -5 & 34 & 34 & 4. & 27 & -21 & -6 & 42 & 49 \\
\hline 1 & 22 & -27 & $\mathrm{~K}, \mathrm{~L}=$ & 86 & 5 & -4 & 15 & 11 & 6 & 20 & 16 & -4 & 37 & -41 \\
\hline 2 & 22 & 25 & -12 & 76 & -81. & -3 & 50 & -54 & $K, L=$ & 7 & 1 & -3 & 23 & -23 \\
\hline 5 & 36 & 38 & -11 & 35 & $39^{\circ}$ & -2 & 33 & -30 & -5 & 16 & -14 & -2 & 34 & 35 \\
\hline 5 & 129 & 30 & -10 & 47 & 43 & 0 & 14 & 17 & -4 & 17 & -10 & -1 & 38 & 36 \\
\hline 6 & 20 & 16 & -5 & 60 & -62 & 1 & 19 & 27 & -3 & 17 & -15 & 1 & 44 & 42 \\
\hline 7 & 47 & -51 & -7 & 17 & -16 & 2 & 108 & -102 & -2 & 29 & -27 & 2 & 21 & 28 \\
\hline 9 & 62 & 770 & -5 & 29 & 32 & 3 & 12 & -16 & -1 & 29 & -24 & $K, L=$ & 7 & 4 \\
\hline$K, L=$ & 6 & 4 & -4 & 48 & 49 & 4 & 30 & 34 & 2 & 38 & -35 & -5 & 20 & 29 \\
\hline-11 & 50 & 60 & -3 & 67 & -70 & 5 & 25 & -36 & 3 & 17 & -18 & -4 & 32 & -33 \\
\hline-8 & 17 & -14 & -1 & 57 & $-57 \cdot$ & $K, L=$ & 6 & $\$ 7$ & 5 & 34 & -32 & -3 & 14 & 12 \\
\hline-7 & 18 & 23 & 0 & 63 & 66 & $\begin{array}{ll}-9 \\
-1\end{array}$ & 10 & -18 & 6 & 43 & -46 & -2 & 15 & -23 \\
\hline-6 & 49 & 58 & 1 & 55 & -52 & -8 & 24 & 24 & $\mathrm{~K}, \mathrm{~L}=$ & 7 & 2 & -1 & 49 & 51 \\
\hline-5 & 19 & 16 & 2 & 54 & -53 & -5 & 13 & 9 & -7 & 30 & 31 & 0 & 14 & -18 \\
\hline-2 & 67 & 61 & 3 & 43 & 47 & -3 & 19 & -13 & -6 & 22 & -28 & & & $\vdots$ \\
\hline$-i$ & 94 & -103 & 5 & 61 & -69 & -2 & 19 & -15 & -5 & 36 & -38 & & & \\
\hline
\end{tabular}


The final themal parameters $\left(210^{4}\right)$ of the non-hydrogon atoms With o.s.d.'s in parentheses

The $\beta_{i j} \mathrm{~s}_{\mathrm{s}}$ are defined by:

$\exp \left(-h^{2} \beta_{11}-k^{2} \beta_{22}-1^{2} \beta_{33}-h k \beta_{12}-h 1 \beta_{13}-k 1 \beta_{23}\right)$.

\begin{tabular}{|c|c|c|c|c|c|c|}
\hline$\therefore$ & $\beta_{11}$ & $\beta_{22}$ & $\beta_{33}$ & $\beta_{12}$ & $\beta_{13}$ & ${ }^{B_{23}}$ \\
\hline$O(1)$ & $22.4(7)$ & $196(6)$ & $156(3)$ & $-4(3)$ & $55(2)$ & $-44(7)$ \\
\hline$O(2)$ & $24,5(6)$ & $270(6)$ & $86(2)$ & $-33(3)$ & $46(2)$ & $-13(6)$ \\
\hline$C(1)$ & $14,5(7)$ & $213(8)$ & $70(2)$ & $4(4)$ & $14(2)$ & $37(7)$ \\
\hline$C(2)$ & $17 \cdot 0(8)$ & $255(9)-$ & $85(3)$ & $-22(4)$ & $17(2)$ & $26(8)$ \\
\hline$C(3)$ & $18.1(8)$ & $333\left(11^{\prime}\right)$ & $113(4)$ & $-26(5)$ & $31(5)$ & $33(10)$ \\
\hline$C(4)$ & $18.8(8)$ & $405(13)$ & $127(4)$ & $22(5)$ & $49(3)$ & $70(11)$ \\
\hline$C(5)$ & $23.4(10)$ & $300(10)$ & $136(4)$ & $25(5)$ & $48(3)$ & $-54(10)$ \\
\hline$C(6)$ & $17.7(8)$ & $227(8)$ & $119(3)$ & $-3(4)$ & $31(3)$ & $-14(9)$ \\
\hline$c(7)$ & $17.8(7)$ & $174(7)$ & $79(3)$ & $-10(4)$ & $36(2)$ & $14(7)$ \\
\hline$C(8)$ & $19.9(8)$ & $214(8)$ & $99(3)$ & $12(4)$ & $23(3)$ & $-26(8)$ \\
\hline$C(9)$ & $25.5(10)$ & $322(11)$ & $102(3)$ & $29(5)$ & $12(3)$ & $22(10)$ \\
\hline$C(10)$ & $28.6(10)$ & $376(12)$ & $79(3)$ & $-26(6)$ & $12(3)$ & $-12(10)$ \\
\hline$C(11)$ & $30.9(11)$ & $289(10)$ & $97(3)$ & $-34(5)$ & $35(3)$ & $-95(9)$ \\
\hline$C(12)$ & $22.4(9)$ & $203(8)$ & $105(3)$ & $-11(4)$ & $38(3)$ & $-18(8)$ \\
\hline$C(13)$ & $16.3(7)$ & $158(7)$ & $79(3)$ & $-2(4)$ & $18(2)$ & $8(7)$ \\
\hline$C(14)^{\prime}$ & $17.6(7)$ & $170(7)$ & $82(3)$ & $-6(4)$ & $30(2)$ & $21(7)$ \\
\hline
\end{tabular}

